\title{
An Efficient Method To Find The Most Influential Parameters of Energy Performance Building Envelopes
}

\author{
Ainagul Jumabekova ${ }^{1}$, Julien Berger ${ }^{1}$, Aurélie Foucquier ${ }^{2}$ \\ ${ }^{1}$ Univ. Grenoble Alpes, Univ. Savoie Mont Blanc, UMR 5271 CNRS, LOCIE, \\ ${ }^{2}$ Univ. Grenoble Alpes, CEA, LITEN, DTS, INES, \\ corresponding author : ainagul.jumabekova@univ-smb.fr
}

\begin{abstract}
This article deals with estimating material properties of existing building walls. Parameter estimation problem generally has a high computational cost due to a large number of direct model evaluations. In this context, a methodology based on derivative based sensitivity analysis is investigated to reduce the number of parameters by identifying the most influential on the wall energy efficiency. The method is applied to estimate parameters impact on the annual thermal loads. It also has a reduced computational cost compared to standard sensitivity analysis approaches. Results demonstrate that the presented method can be extended to more complex model equations.
\end{abstract}

\section{Introduction}

The building sector is one of the main contributors to total worldwide energy (EIA (2015)). The energy consumption of buildings in the $\mathrm{EU}$ is about $40 \%$ of the total demand. Nevertheless, efficient insulation of the building envelope contributes to energy savings. While choosing the optimal retrofitting actions engineers mostly rely on building energy simulation programs. However, existing buildings have several uncertainties which imply the lack of reliability of the simulations. The knowledge of the thermophysical properties of the wall materials is one of them. In order to minimise these uncertainties, the current wall material properties should be identified through the solution of an inverse problem.

A parameter estimation problem has often a high computational cost since it is proportional to the number of model parameters. Therefore, it is essential to find out, which parameters have more effect on the model output and eliminate non-significant ones. Using the sensitivity coefficients, which calculation is required in the context of the parameter estimation problem, it allows us to highlight the parameters which most influence the outputs. Thus, in the framework of the parameter estimation problem, a preliminary step is proposed and denoted as Primary Identifiability. This procedure implies ranking all the model parameters regarding their impact on the model output and removing the insignificant and correlated model parameters. Under model output, we mean any characteristic of building energy behaviour, for example, the wall temperature, the wall heat flux or the thermal loads. The methodology is schematically synthesized in Figure 1. The Primary Identifiability approach relies on the sensitivity coefficients, so the purpose is now to determine their calculation.

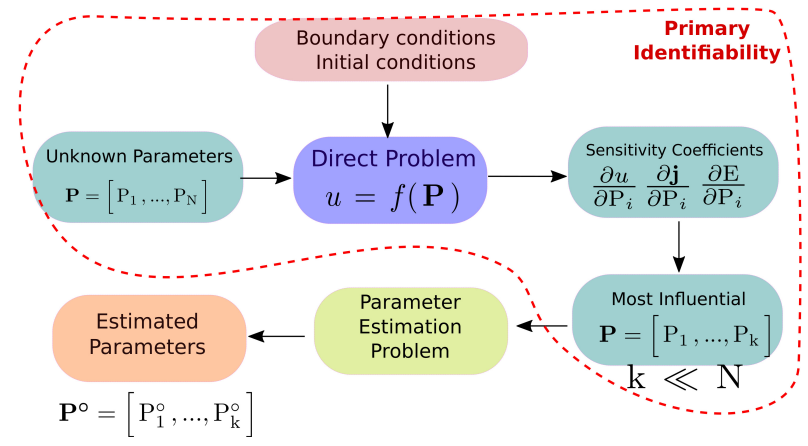

Figure 1: Methodology of Primary Identifiability.

There have been many research papers, which study various sensitivity analysis techniques, including local and global approaches. The screening methods are generally used to determine the scope of the most valuable parameters of building energy model (Menberg et al. (2016)) due to its comparatively small computational cost. However, these methods are qualitative and cannot quantify the variation of the model output relative to the change of the input parameter. Several methods for local and global sensitivity analysis can provide the sensitivity coefficients values. The local approach estimates the parameter impact only at one point of the parameter domain without providing the detailed information. It was used to explore the parameter influence (Barrio and Guyon (2003)). Global methods consider the whole spectrum of the parameter space and some of them reveal the interaction between parameters. However, examples of regression-based (Sun et al. (2014); Wilde and Tian (2010)) and variance-based techniques (Ruiz Flores et al. (2012); Mechri et al. (2010)) show that these methods require an extensive number of model eval- 
uations, and therefore can not be used as a prior step for a parameter estimation problem.

On the other hand, the direct differentiation of the model governing equation enables to retrieve values of sensitivity coefficients without countless model simulations. To our knowledge, this approach has never been explored for building energy simulations. By taking into account second order derivatives, the combinatorial influences can also be captured in this approach. The effort of solving the additional sensitivity equations prevents this method from widespread implementation. Our idea is to use DUFORT-FrANKEL numerical scheme to numerically solve these equations. This innovative numerical model has benefits of being unconditionally stable, second-order accurate in time and having an explicit formulation (Gasparin et al., 2018a). This approach allows us retrieving values of sensitivity coefficients with higher accuracy at a low computational cost. Additionally, obtained sensitivity coefficients are defined continuously over space and time domains.

Thus, the objective of this paper is to validate our methodology. For this, a base case scenario of heat conduction transfer through a wall is considered. Then, the subset of parameters which have more influence on the heat flux and thermal loads are identified. The efficiency of the method is evaluated.

The article is organized in the following way. At first, the physical model is defined together with its sensitivity equations. Then, the model output extrapolation through the TAYLOR series expansion is detailed. The numerical solution of the sensitivity coefficients using the DUFORT-FRANKEL numerical scheme is introduced. Next, the case study of a building wall is described. Finally, the results of parameter influence on the heat flux and the thermal loads, its comparison with the linear regression techniques are demonstrated.

\section{Methods}

\section{Physical model}

Let us consider the one-dimensional heat conduction transfer operating in a building wall. The wall is composed of $N$ layers with different thicknesses and thermal properties. Figure 2 illustrates the problem. The governing transient heat conduction equation is as follows:

$$
c \frac{\partial T}{\partial t}=\frac{\partial}{\partial x}\left(k \frac{\partial T}{\partial x}\right)
$$

where $T[\mathrm{~K}]$ is the temperature defined on the domains $\Omega_{x}: x \in[0, L]$ and $\Omega_{t}: t \in\left[0, \tau_{\max }\right], L[\mathrm{~m}]$ is the length of the wall. Both the volumetric heat capacity $c\left[\mathrm{~J} /\left(\mathrm{m}^{3} \cdot \mathrm{K}\right)\right]$ and the thermal conductivity $k[\mathrm{~W} /(\mathrm{m} \cdot \mathrm{K})]$ depend on space according to the fol-

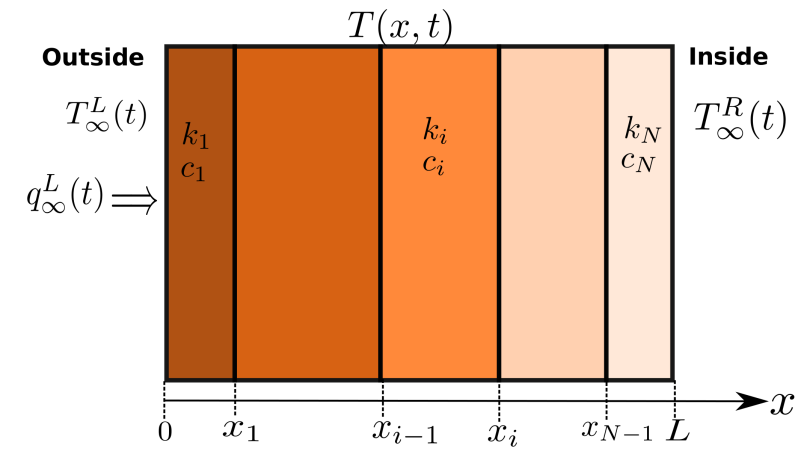

Figure 2: Illustration of the heat transfer through the wall.

lowing equations:

$$
c=\sum_{i=1}^{N} c_{i} \cdot \varphi_{i}(x), \quad k=\sum_{i=1}^{N} k_{i} \cdot \varphi_{i}(x),
$$

where functions $\varphi_{i}(x)$ are defined as:

$$
\varphi_{i}(x)= \begin{cases}1, & x_{i-1} \leqslant x \leqslant x_{i} \\ 0, & \text { otherwise }\end{cases}
$$

So our equation (1) becomes:

$$
\sum_{i=1}^{N} c_{i} \cdot \varphi_{i}(x) \cdot \frac{\partial T}{\partial t}=\frac{\partial}{\partial x}\left(\sum_{i=1}^{N} k_{i} \cdot \varphi_{i}(x) \cdot \frac{\partial T}{\partial x}\right)
$$

The outside part is exposed to weather conditions and solar radiation. The inside wall surface is in contact with the room air temperature. Therefore, let us assume RoBIn boundary conditions as:

$$
\begin{aligned}
\left.k \frac{\partial T}{\partial x}\right|_{x=0} & =h_{L}\left(T(0, t)-T_{\infty}^{L}(t)\right)-\alpha q_{\infty}^{L}(t) \\
\left.k \frac{\partial T}{\partial x}\right|_{x=L} & =-h_{R}\left(T(L, t)-T_{\infty}^{R}(t)\right),
\end{aligned}
$$

where $T_{\infty}^{L}(t)[\mathrm{K}]$ and $T_{\infty}^{R}(t)[\mathrm{K}]$ are temperature of the inside and outside air respectively, which vary over the time, $\mathrm{h}_{\mathrm{L}}\left[\mathrm{W} /\left(\mathrm{m}^{2} \cdot \mathrm{K}\right)\right]$ and $\mathrm{h}_{\mathrm{R}}\left[\mathrm{W} /\left(\mathrm{m}^{2} \cdot \mathrm{K}\right)\right]$ are inside and outside surface convective heat transfer coefficients, and $q_{\infty}^{L}(t)\left[\mathrm{W} / \mathrm{m}^{2}\right]$ is the total solar radiation, which includes the direct, diffuse and reflexive radiations, and $\alpha$ is the surface absorptivity.

At the initial state the temperature is given as:

$$
T(x, t=0)=T_{0}(x)
$$

As mentioned before, model outputs are the heat flux, induced on the wall, and the thermal loads.

The heat flux $\mathrm{j}\left(x^{\circ}, t\right)\left[\mathrm{W} / \mathrm{m}^{2}\right]$ is described by FOURIER's law:

$$
\mathrm{j}\left(x^{\circ}, t\right)=-\left.k\left(x^{\circ}\right) \frac{\partial T}{\partial x}\right|_{x=x^{\circ}}, x^{\circ} \in\{0, L\}
$$


The thermal loads $\mathrm{E}\left(x^{\circ}, t\right)\left[\mathrm{W} \cdot \mathrm{h} / \mathrm{m}^{2}\right]$ for a time period between $t_{1}$ and $t_{2}$ are calculated by the following expression:

$\mathrm{E}\left(x^{\circ}, t\right)=-\int_{t_{1}}^{t_{2}} k\left(x^{\circ}\right) \frac{\partial T}{\partial x}\left(x^{\circ}, \tau\right) \mathrm{d} \tau, x^{\circ} \in\{0, L\}$

\section{Dimensionless formulation}

In order to minimize the round-off numerical errors and to analyze the model behaviour regardless the used units for variables, it is essential to obtain a dimensionless formulation of the problem (Berger et al. (2017); Trabelsi et al. (2018)). To convert equation (1) let us introduce the following dimensionless variables:

$$
x^{\star}=\frac{x}{L}, \quad u=\frac{T}{T_{\mathrm{ref}}}, \quad t^{\star}=\frac{t}{t_{\mathrm{ref}}},
$$

as well as dimensionless thermal properties functions by:

$$
\begin{aligned}
& k^{\star}: \stackrel{\text { def }}{=} \frac{k}{k_{\text {ref }}}, \quad \text { or } \quad k^{\star}=\sum_{i=1}^{N} \frac{k_{i}}{k_{\text {ref }}} \cdot \varphi_{i}\left(x^{\star}\right), \\
& c^{\star}: \stackrel{\text { def }}{=} \frac{c}{c_{\text {ref }}}, \quad \text { or } \quad c^{\star}=\sum_{i=1}^{N} \frac{c_{i}}{c_{\text {ref }}} \cdot \varphi_{i}\left(x^{\star}\right) .
\end{aligned}
$$

where subscripts ref relate for a characteristic reference value, and superscript $\star$ for dimensionless parameters.

Thus, equation (1) transforms to:

$$
c^{\star} \frac{\partial u}{\partial t^{\star}}=\operatorname{Fo} \frac{\partial}{\partial x}\left(k^{\star} \frac{\partial u}{\partial x^{\star}}\right),
$$

where Fo $=\frac{t_{\text {ref }} k_{\text {ref }}}{L^{2} c_{\text {ref }}}$ is FOURIER number.

The RoBIN-type boundary conditions are converted to:

$$
\begin{array}{rlrl}
k^{\star} \frac{\partial u}{\partial x^{\star}} & =\mathrm{Bi}_{\mathrm{L}}\left(u-u_{\infty}^{\mathrm{L}}\right)-\alpha g_{\infty}^{\mathrm{L}}, & & x^{\star}=0 \\
k^{\star} \frac{\partial u}{\partial x^{\star}} & =-\mathrm{Bi}_{\mathrm{R}}\left(u-u_{\infty}^{\mathrm{R}}\right), & x^{\star}=1,
\end{array}
$$

with dimensionless quantities

$$
\begin{aligned}
& \mathrm{Bi}_{\mathrm{L}}=\frac{\mathrm{h}_{\mathrm{L}} L}{k_{\text {ref }}}, \quad \mathrm{Bi}_{\mathrm{R}}=\frac{\mathrm{h}_{\mathrm{R}} L}{k_{\text {ref }}}, \\
& u_{\infty}^{\mathrm{L}}:=\frac{T_{\infty}^{\mathrm{L}}}{T_{\text {ref }}}, \quad u_{\infty}^{\mathrm{R}}:=\frac{T_{\infty}^{\mathrm{d}}}{T_{\text {ref }}}, \quad g_{\infty}^{\mathrm{L}} \stackrel{\text { def }}{=} \frac{q_{\infty}^{\mathrm{L}} L}{T_{\text {ref }} k_{\text {ref }}} .
\end{aligned}
$$

The uniform initial condition transforms to

$$
u=u_{0}, \quad \text { where } \quad u_{0} \stackrel{\text { def }}{=} \frac{T_{0}}{T_{\text {ref }}}
$$

The dimensionless heat flux $\mathrm{j}^{\star}$ is expressed as:

$\mathrm{j}^{\star}\left(x_{s}^{\star}, t^{\star}\right)=\left.\left(-k^{\star} \frac{\partial u}{\partial x^{\star}}\right)\right|_{x^{\star}=x_{s}^{\star}}, x_{s}^{\star} \in\{0,1\}$
The dimensionless thermal loads $\mathrm{E}^{\star}$ are calculated as:

$$
\mathrm{E}^{\star}\left(t^{\star}\right)=\int_{t_{1}^{\star}}^{t_{2}^{\star}} \mathrm{j}^{\star}\left(x_{s}^{\star}, \tau^{\star}\right) \mathrm{d} \tau^{\star} .
$$

Further in the article, for the sake of the clarity the superscript $\star$ is omitted, and all results are presented in dimensionless form unless stated otherwise.

\section{Sensitivity equations}

Let us consider solution $u$ of Eq. (7) as a model output. The solution $u$ may be declared as a function of $(x, t, \mathrm{P})$, where $\mathrm{P}$ is a set of model parameters. In our case one may formulate is as follows:

$$
u:(x, t, \mathrm{P}) \longmapsto u(x, t, \mathrm{P}),
$$

where $\mathrm{P}=\left\{k_{i}, c_{i}\right\}, \forall i=1 \ldots N$

Let us introduce new variables $X_{P_{i}}$ or sensitivity coefficients, which quantify the model output sensitivity to the parameter $P_{i}$. A parameter is called sensitive if small changes in its value cause large changes in the model output.

$$
X_{P_{i}} \stackrel{\text { def }}{=} \frac{\partial u}{\partial P_{i}}
$$

The sensitivity coefficients are obtained as a solution of a differential equation or sensitivity equation, which is a result of partial differentiation of the model equation. Therefore, differential sensitivity analysis has the advantage to retrieve sensitivity coefficients accurately and continuously defined on space and time domains.

For the sake of simplicity, let us find sensitivity equations for the first layer. The following new variables are presented:

$$
X_{k_{1}}: \stackrel{\text { def }}{=} \frac{\partial u}{\partial k_{1}}, \quad X_{c_{1}} \stackrel{\text { def }}{:} \frac{\partial u}{\partial c_{1}} .
$$

Let us differentiate Eq. (7) with respect to parameter $k_{1}$. We obtain the differential equation for $X_{k_{1}}$ :

$$
\frac{\partial X_{k_{1}}}{\partial t}=\frac{\text { Fo }}{c} \frac{\partial}{\partial x}\left(\frac{\partial k}{\partial k_{1}} \frac{\partial u}{\partial x}+k \frac{\partial X_{k_{1}}}{\partial x}\right)
$$

Similarly, sensitivity equation is obtained for $X_{c_{1}}$ :

$$
\begin{aligned}
& \frac{\partial X_{c_{1}}}{\partial t}=-\frac{\text { Fo }}{c^{2}} \frac{\partial c}{\partial c_{1}} \frac{\partial}{\partial x}\left(k \frac{\partial u}{\partial x}\right)+ \\
& \frac{\text { Fo }}{c} \frac{\partial}{\partial x}\left(k \frac{\partial X_{c_{1}}}{\partial x}\right)
\end{aligned}
$$

The second order differentiation of Eq. (7) gives us expressions for following variables:

$$
X_{k_{1} k_{1}} \stackrel{\text { def }}{=} \frac{\partial^{2} u}{\partial k_{1}{ }^{2}}, X_{c_{1} c_{1}} \stackrel{\text { def }}{=} \frac{\partial^{2} u}{\partial c_{1}^{2}}, X_{k_{1} c_{1}} \stackrel{\text { def }}{:=} \frac{\partial^{2} u}{\partial k_{1} \partial c_{1}} \text {. }
$$


The variable $X_{k_{1} c_{1}}$ describes the change in model output with respect to two varying parameters. It is computed as a solution to the following equation:

$$
\begin{aligned}
\frac{\partial X_{k_{1} c_{1}}}{\partial t}= & -\frac{\mathrm{Fo}}{c^{2}} \frac{\partial c}{\partial c_{1}} \frac{\partial}{\partial x}\left(\frac{\partial k}{\partial k_{1}} \frac{\partial u}{\partial x}+k \frac{\partial X_{k_{1}}}{\partial x}\right)+ \\
& \frac{\text { Fo }}{c} \frac{\partial}{\partial x}\left(\frac{\partial k}{\partial k_{1}} \frac{\partial X_{c_{1}}}{\partial x}+k \frac{\partial X_{k_{1} c_{1}}}{\partial x}\right)
\end{aligned}
$$

The defined above sensitivity coefficients are used to calculate how the variation of the model parameters will impact other model outputs such as heat flux and thermal loads. Let us demonstrate expressions for sensitivity coefficients of the heat flux on the outside surface.

$$
\begin{aligned}
\frac{\partial \mathrm{j}}{\partial k_{1}}(0, t) & =-\frac{\partial u}{\partial x}-k_{1} \frac{\partial X_{k_{1}}}{\partial x} \\
\frac{\partial \mathrm{j}}{\partial c_{1}}(0, t) & =-k_{1} \frac{\partial X_{c_{1}}}{\partial x} \\
\frac{\partial^{2} \mathrm{j}}{\partial k_{1}^{2}}(0, t) & =-2 \frac{\partial X_{k_{1}}}{\partial x}-k_{1} \frac{\partial X_{k_{1} k_{1}}}{\partial x} \\
\frac{\partial^{2} \mathrm{j}}{\partial c_{1}^{2}}(0, t) & =-k_{1} \frac{\partial X_{c_{1} c_{1}}}{\partial x}, \\
\frac{\partial^{2} \mathrm{j}}{\partial k_{1} \partial c_{1}}(0, t) & =-\frac{\partial X_{c_{1}}}{\partial x}-k_{1} \frac{\partial X_{k_{1} c_{1}}}{\partial x}
\end{aligned}
$$

\section{Metrics for the sensitivity coefficients}

Let us introduce an estimator of the individual parameter importance, which is defined below:

$$
\eta_{P_{i}}=\frac{\int_{\Omega_{x}}\left(\int_{\Omega_{t}} X_{P_{i}}(\chi, \tau) \mathrm{d} \tau\right)^{2} \mathrm{~d} \chi}{\sum_{r=1}^{N} \int_{\Omega_{x}}\left(\int_{\Omega_{t}} X_{P_{r}}(\chi, \tau) \mathrm{d} \tau\right)^{2} \mathrm{~d} \chi},
$$

where $X_{P_{i}}$ is sensitivity coefficient of the parameter $P_{i}$ on the chosen model output obtained through the solution of the corresponding sensitivity equation, and $N$ is the total number of the input parameters which influence on the output.

A large value of this metric means high influence on the output, while small one indicates nonsensitive parameters.

\section{Taylor series expansion}

Knowledge of the partial derivatives facilitates the approximation of the model output by using the TAYLOR series. Indeed, since we are in the context of parameter estimation problem, the model output can be represented in the neighbourhood of the a priori values of parameters. The following expression describes the estimation of the function $u\left(x, t, k_{1}, c_{1}\right)$ at every point of the parameters $\left\{k_{1}, c_{1}\right\}$ around the a priori parameters values $\left\{k_{1}^{\circ}, c_{1}^{\circ}\right\}$.

$$
\begin{aligned}
& u\left(x, t, k_{1}, c_{1}\right)=u\left(x, t, k_{1}^{\circ}, c_{1}^{\circ}\right)+ \\
& \left.\frac{\partial u}{\partial k_{1}}\right|_{k_{1}=k_{1}^{\circ}}\left(k_{1}-k_{1}^{\circ}\right)+\left.\frac{\partial u}{\partial c_{1}}\right|_{c_{1}=c_{1}^{\circ}}\left(c_{1}-c_{1}^{\circ}\right)+ \\
& \left.\frac{1}{2} \frac{\partial^{2} u}{\partial k_{1}^{2}}\right|_{k_{1}=k_{1}^{\circ}}\left(k_{1}-k_{1}^{\circ}\right)^{2}+\left.\frac{1}{2} \frac{\partial^{2} u}{\partial c_{1}^{2}}\right|_{c_{1}=c_{1}^{\circ}}\left(c_{1}-c_{1}^{\circ}\right)^{2}+
\end{aligned}
$$

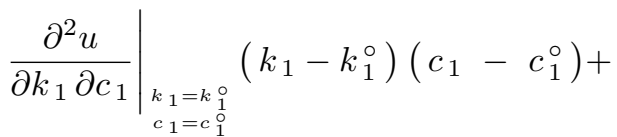

$$
\begin{aligned}
& \mathcal{O}\left(\max \left\{\left(k_{1}-k_{1}^{\circ}\right)^{3},\left(c_{1}-c_{1}^{\circ}\right)^{3}\right\}\right) .
\end{aligned}
$$

Similarly, second order TAYLOR approximation is defined for the thermal loads on the exterior surface as a model output.

$$
\begin{aligned}
& E\left(0, t, k_{1}, c_{1}\right)=E\left(0, t, k_{1}^{\circ}, c_{1}^{\circ}\right)+ \\
& \left.\frac{\partial E}{\partial k_{1}}\right|_{k_{1}=k_{1}^{\circ}}\left(k_{1}-k_{1}^{\circ}\right)+\left.\frac{\partial E}{\partial c_{1}}\right|_{c_{1}=c_{1}^{\circ}}\left(c_{1}-c_{1}^{\circ}\right)+ \\
& \left.\frac{1}{2} \frac{\partial^{2} E}{\partial k_{1}^{2}}\right|_{k_{1}=k_{1}^{\circ}}\left(k_{1}-k_{1}^{\circ}\right)^{2}+\left.\frac{1}{2} \frac{\partial^{2} E}{\partial c_{1}^{2}}\right|_{c_{1}=c_{1}^{\circ}}\left(c_{1}-c_{1}^{\circ}\right)^{2}+
\end{aligned}
$$

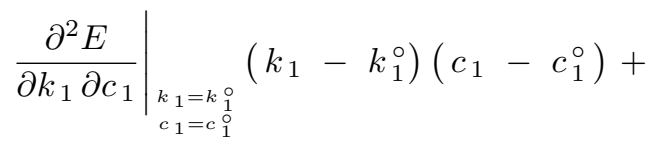

$$
\begin{aligned}
& \mathcal{O}\left(\max \left\{\left(k_{1}-k_{1}^{\circ}\right)^{3},\left(c_{1}-c_{1}^{\circ}\right)^{3}\right\}\right) .
\end{aligned}
$$

The expressions above are a powerful tool to explore how the model output value changes while varying the parameters of the model.

\section{Numerical solution}

Let us discretize uniformly the space $\Omega_{x}$ and time $\Omega_{t}$ intervals, with the parameters $\Delta x$ and $\Delta t$, respectively. The discrete values of function $u(x, t)$ are defined as $u_{j}^{n} \stackrel{\text { def }}{=} u\left(x_{j}, t_{n}\right)$, where $j \in\left\{1, \ldots, N_{x}\right\}$ and $n \in\left\{1, \ldots, N_{t}\right\}$.

Numerical solution of the Eq. (7) is obtained using DufOrT-Frankel scheme (Gasparin et al. (2018b)). As previously mentioned, this numerical scheme allows to compute explicitly the solution at each time step, and it has the unconditionally stable property. The numerical scheme can be expressed as the following explicit formulation:

$$
u_{j}^{n+1}=\nu_{1} u_{j+1}^{n}+\nu_{2} u_{j-1}^{n}+\nu_{3} u_{j}^{n-1},
$$

where

$$
\begin{aligned}
\nu_{1} & =\frac{\lambda_{1}}{\lambda_{0}+\lambda_{3}}, \quad \nu_{2}=\frac{\lambda_{2}}{\lambda_{0}+\lambda_{3}} \\
\nu_{3} & =\frac{\lambda_{0}-\lambda_{3}}{\lambda_{0}+\lambda_{3}}
\end{aligned}
$$


and

$$
\begin{aligned}
& \lambda_{0}=1, \quad \lambda_{3}=\frac{\Delta t}{\Delta x^{2}} \frac{\mathrm{Fo}}{c_{j}}\left(k_{j+\frac{1}{2}}+k_{j-\frac{1}{2}}\right), \\
& \lambda_{1}=\frac{2 \Delta t}{\Delta x^{2}} \frac{\mathrm{Fo}}{c_{j}} k_{j}+\frac{1}{2}, \quad \lambda_{2}=\frac{2 \Delta t}{\Delta x^{2}} \frac{\mathrm{Fo}}{c_{j}} k_{j-\frac{1}{2}}, \\
& k_{j \pm \frac{1}{2}}=k\left(\frac{x_{j}+x_{j \pm 1}}{2}\right) .
\end{aligned}
$$

In order to calculate the sensitivity coefficients efficiently, the direct differentiation of the numerical scheme Eq. (14) with respect to the required parameter is used.

One may obtain the numerical scheme for computing sensitivity coefficient $X_{k_{1}}$ of the model output $u(x, t)$ with a respect to a parameter $k_{1}$, by partially differentiation of each term of Eq. (14), which is presented below:

$$
\begin{aligned}
X_{k_{1_{j}}}^{n+1}= & \nu_{1} X_{k_{1_{j+1}}}^{n}+\nu_{2} X_{k_{1_{j}-1}}^{n}+\nu_{3} X_{k_{1_{j}}}^{n-1}+ \\
& \frac{\partial \nu_{1}}{\partial k_{1}} u_{j+1}^{n}+\frac{\partial \nu_{2}}{\partial k_{1}} u_{j-1}^{n}+\frac{\partial \nu_{3}}{\partial k_{1}} u_{j}^{n-1}
\end{aligned}
$$

Similarly, expression for sensitivity coefficient $X_{c_{1}}$ is as follows:

$$
\begin{aligned}
X_{c_{1} j}^{n+1}= & \nu_{1} X_{c_{1_{j+1}}}^{n}+\nu_{2} X_{c_{1_{j}-1}}^{n}+\nu_{3} X_{c_{1_{j}}}^{n-1}+ \\
& \frac{\partial \nu_{1}}{\partial c_{1}} u_{j+1}^{n}+\frac{\partial \nu_{2}}{\partial c_{1}} u_{j-1}^{n}+\frac{\partial \nu_{3}}{\partial c_{1}} u_{j}^{n-1}
\end{aligned}
$$

Second order partial differentiation of Eq. (14) with respect to parameter $k_{1}$ results as numerical scheme for $X_{k_{1} k_{1}}$ :

$$
\begin{array}{r}
X_{k_{1} k_{1_{j}}}^{n+1}=\nu_{1} X_{k_{1} k_{1_{j+1}}}^{n}+\nu_{2} X_{k_{1} k_{1_{j-1}}}^{n}+\nu_{3} X_{k_{1} k_{1_{j}}}^{n-1}+ \\
2 \frac{\partial \nu_{1}}{\partial k_{1}} X_{k_{1_{j+1}}}^{n}+2 \frac{\partial \nu_{2}}{\partial k_{1}} X_{k_{1_{j-1}}}^{n}+2 \frac{\partial \nu_{3}}{\partial k_{1}} X_{k_{1_{j}}}^{n-1}+ \\
\frac{\partial^{2} \nu_{1}}{\partial k_{1}^{2}} u_{j+1}^{n}+\frac{\partial^{2} \nu_{2}}{\partial k_{1}^{2}} u_{j-1}^{n}+\frac{\partial^{2} \nu_{3}}{\partial k_{1}^{2}} u_{j}^{n-1}
\end{array}
$$

The variation of model output over both parameters $k_{1}$ and $c_{1}$ is given through the numerical scheme for $X_{k_{1} c_{1}}$ and given below:

$$
\begin{array}{r}
X_{k_{1} c_{1} j}^{n+1}=\nu_{1} X_{k_{1} c_{1_{j}+1}}^{n}+\nu_{2} X_{k_{1} c_{1_{j}-1}}^{n}+\nu_{3} X_{k_{1} c_{1_{j}}}^{n-1}+ \\
\frac{\partial \nu_{1}}{\partial c_{1}} X_{k_{1_{j}+1}}^{n}+\frac{\partial \nu_{2}}{\partial c_{1}} X_{k_{1_{j}-1}}^{n}+\frac{\partial \nu_{3}}{\partial k_{1}} X_{k_{1_{j}}}^{n-1}+ \\
\frac{\partial \nu_{1}}{\partial k_{1}} X_{c_{1_{j+1}}}^{n}+\frac{\partial \nu_{2}}{\partial k_{1}} X_{c_{1_{j-1}}}^{n}+\frac{\partial \nu_{3}}{\partial k_{1}} X_{c_{1_{j}}}^{n-1}+ \\
\frac{\partial^{2} \nu_{1}}{\partial k_{1} \partial c_{1}} u_{j+1}^{n}+\frac{\partial^{2} \nu_{2}}{\partial k_{1} \partial c_{1}} u_{j-1}^{n}+\frac{\partial^{2} \nu_{3}}{\partial k_{1} \partial c_{1}} u_{j}^{n-1}
\end{array}
$$

\section{Case study}

In this section, the proposed approach is investigated to determine the parameters which most influence the energy performance of a building wall. The latter is located in Omsk city in the north region of Russia. The wall is composed of four materials: gypsum board, clay brick, an insulation layer (mineral wool) and the cement sandy solution. This structure, illustrated in Figure 3, is commonly used in Russia. The thermal properties of materials are given below according to (Franchuk (1969), Kononovich (1986)).

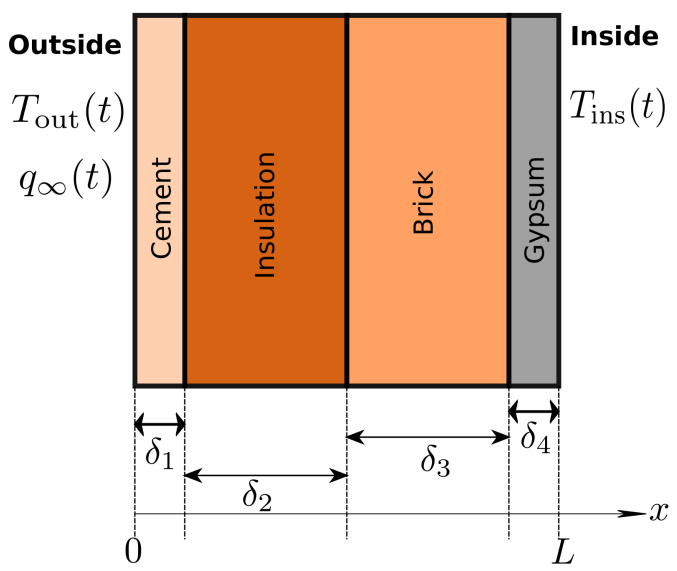

Figure 3: Illustration of wall composition.

$$
\begin{array}{clc}
\delta[\mathrm{m}] & k[\mathrm{~W} / \mathrm{m} \cdot \mathrm{K}] & c\left[\mathrm{~J} / \mathrm{m}^{3} \cdot \mathrm{K}\right] \\
\delta_{1}=0.03, & k_{1}=1.4, & c_{1}=1.84 \times 10^{6}, \\
\delta_{2}=0.19, & k_{2}=0.043, & c_{2}=0.08 \times 10^{6}, \\
\delta_{3}=0.38, & k_{3}=0.52, & c_{3}=1.23 \times 10^{6}, \\
\delta_{4}=0.02, & k_{4}=0.17, & c_{4}=0.87 \times 10^{6} .
\end{array}
$$

Outside temperature $T_{\text {out }}(t)$ is obtained through historical weather data. Inside temperature $T_{\text {ins }}(t)$ is set as periodic function, which changes from $18^{\circ} \mathrm{C}$ to $25^{\circ} \mathrm{C}$. Annual time profiles of exterior and interior air temperatures are presented in Figure 4 . The outside and inside surface convective heat transfer coefficients are to set as $h_{\text {out }}=15\left[\mathrm{~W} / \mathrm{m}^{2} \cdot \mathrm{K}\right]$ and $h_{\text {ins }}=8\left[\mathrm{~W} / \mathrm{m}^{2} \cdot \mathrm{K}\right]$ respectively. Daily solar radiation is interpolated hourly in Figure 5. In order to avoid influence of the initial condition the temperature $T_{0}(x)$ is calculated as a solution of the steady state problem.

Considering all this setup, the temperature inside the wall is computed over one year together with the sensitivity coefficients for each thermal parameter of each layer. Their influence on each model output is then calculated. Next section presents these results.

\section{Results and Discussions}

Table 1 gives the parameter impact estimator on the temperature $T$ within each layer. As one may conclude, the influence of the thermal conductivity is higher than the volumetric thermal capacity. Indeed, the thermal capacity influence is almost negligible. Therefore, this parameter can be eliminated in the perspective of a parameter estimation procedure. 


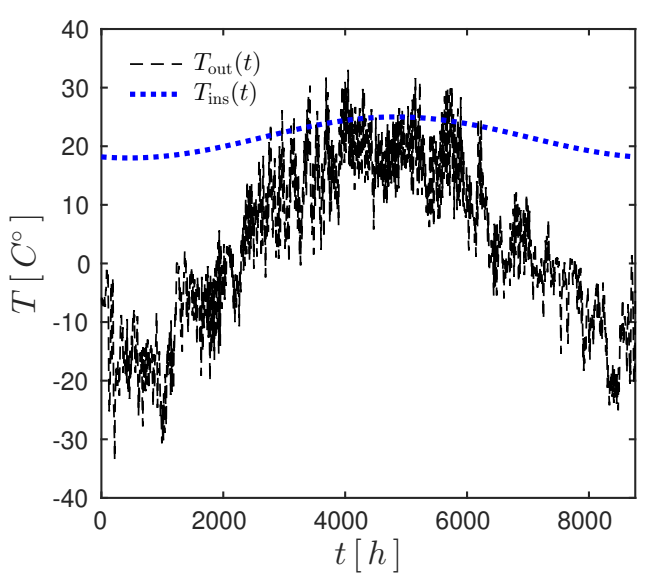

Figure 4: Temperature profiles on the boundaries.

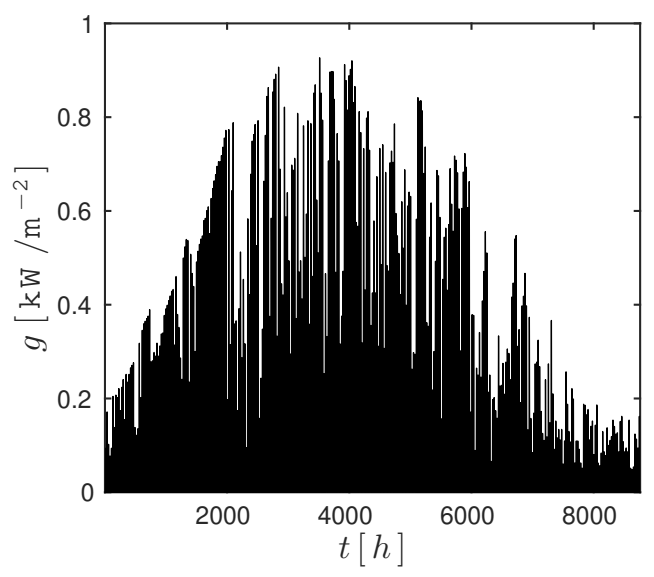

Figure 5: Solar radiation on the wall.

Table 2 presents the importance factor of each parameter on the thermal loads $E$ computed on the inside surface. As previously we can observe that the thermal conductivity is the most influential parameter.

\section{Comparison with Standardized Regression} Coefficients

A brief comparison with standard approach from literature, namely the Standard Regression Coefficient (SRC), is now performed. For this, it is assumed that the model output is linearly dependent on the parameter set. The thermal loads are chosen as model output. Thus, the thermal loads are expressed as:

$$
E=\beta_{i} p_{i}+\beta_{0}, i=\{1, \ldots, M\},
$$

where $\beta_{i}$ is a regression coefficient, $\beta_{0}-$ constant, and $p_{i}$ - parameters of the model, $M$ - total number of parameters. The Standard Regression Coefficient (SRC) $\mathrm{S}_{i}$ is defined as follows:

$$
\mathrm{S}_{i}=\beta_{i} \sqrt{\frac{\operatorname{Var}\left(p_{i}\right)}{\operatorname{Var}(E)}}, i=\{1, \ldots, M\}
$$

which under some assumptions can be used as sensitivity coefficients (Saltelli et al. (2004)).

The SRC coefficients for the thermal conductivity and the heat capacity for the brick layer are now computed. A Latin Hypercube sampling is carried out to
Table 1: Parameter impact estimator $\eta_{P_{i}}$ on the temperature $T$.

\begin{tabular}{|c|c|c|c|c|}
\hline & Gypsum & Brick & Insulation & Cement \\
\hline$k_{i}^{\star}$ & 1 & 0.99 & 1 & 0.9978 \\
\hline$c_{i}^{\star}$ & $4.7 \mathrm{e}-11$ & $2.8 \mathrm{e}-04$ & $1.9 \mathrm{e}-06$ & $2.2 \mathrm{e}-03$ \\
\hline
\end{tabular}

Table 2: Parameter impact estimator $\eta_{P_{i}}$ on the thermal loads E.

\begin{tabular}{|c|c|c|c|c|}
\hline & Gypsum & Brick & Insulation & Cement \\
\hline$k_{i}^{\star}$ & 1 & 0.9532 & 1 & 0.99 \\
\hline$c_{i}^{\star}$ & $1.98 \mathrm{e}-12$ & 0.0468 & $4.6 \mathrm{e}-07$ & $4.22 \mathrm{e}-04$ \\
\hline
\end{tabular}

generate $N_{\mathrm{s}}$ samples between $20 \%$ ratio of the a prior $i$ values of parameters $k_{3}$ and $c_{3}$. For each sample, the model output is computed. Then, a Lasso regression method is used to determine the regression coefficients $\beta_{i}$. Finally, the SRC coefficients values are obtained and the importance factor are computed according to:

$$
\nu_{p_{i}}=\frac{\mathrm{S}_{i}^{2}}{\sum_{m=1}^{M} \mathrm{~S}_{m}^{2}}
$$

Results are reported in Table 3. As one may note, a minimal number of model evaluations is required to compute the SRC coefficients. The importance factor values do not vary with the increases for $N_{\mathrm{s}} \geqslant 10$, validating the convergence of the approach.

Table 3: Parameter importance factor within the brick layer, using SRC coefficients.

\begin{tabular}{|c|c|c|c|c|}
\hline$N_{\mathbf{s}}$ & 5 & 10 & 20 & 50 \\
\hline$\nu_{k_{3}}$ & - & 0.9207 & 0.9266 & 0.9221 \\
\hline$\nu_{c_{3}}$ & - & 0.0793 & 0.0734 & 0.0779 \\
\hline
\end{tabular}

Results obtained with the SRC approach are similar to the ones obtained with the direct differentiation method. It validates the robustness of the differential sensitivity approach. The performance of both methods can be analyzed. The determination of the SRC coefficients depends on the parameter sampling. In this case, at least $N_{\mathrm{s}}=10$ model evaluations are required. The direct differentiation only requires 5 model evaluation for two input parameters. Indeed, this interpretation comes directly from Eq. (15), (16). Each first-order partial derivative requires twice as many operations than the model output.

In terms of computational time, the computation of the sensitivity coefficients through the differential sensitivity analysis requires approximately $30 \mathrm{~min}-$ utes. For the SRC coefficients, the computation takes 1 hour for $N_{\mathrm{s}}=10$ samples.

\section{Application of Taylor series expansion}

One of the main advantages of the direct differentiation approach is to explore the model output on the neighborhood of the a priori parameter values. Us- 
ing sensitivity coefficients and TAYLOR series expansion one may evaluate the influence of the thermal parameters on the model output. Since the volumetric heat capacity has almost no impact on the heat flux, the analysis is carried out with respect to the thermal thermal conductivity of the insulation layer. The value of parameter $k_{2}$ is varied by $\pm 50 \%$ of its a priori value. Then, the heat flux is interpolated for $0.5 \cdot k_{2}^{\circ}$ and for $1.5 \cdot k_{2}^{\circ}$ using the TAYLOR series approximation. Since the direct differentiation approach enables to work with output model depending on time or space, it is possible to evaluate the time variation of the heat flux during the whole year for the boundary values of $k_{2}$ as shown in Figure 6 .

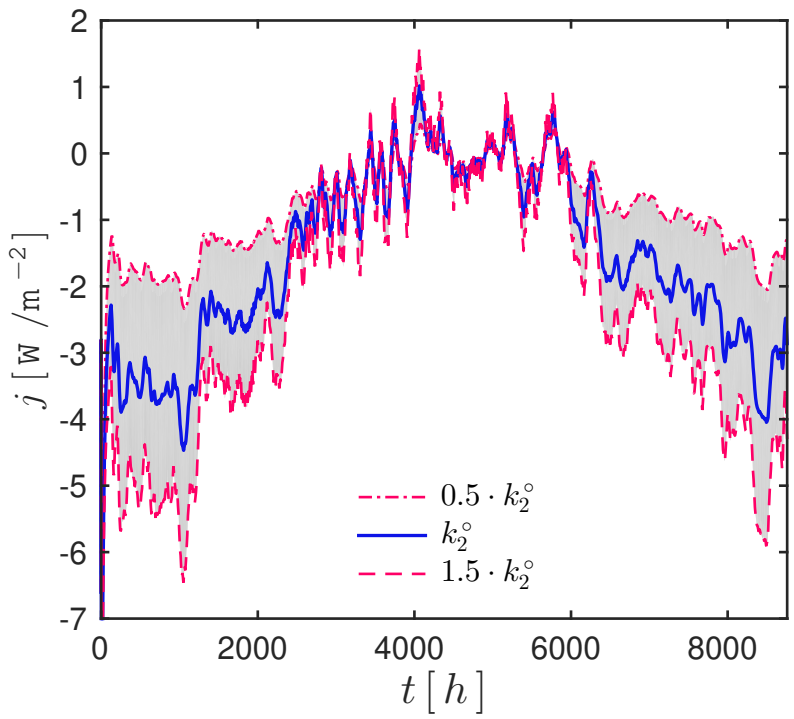

Figure 6: Extrapolation of the heat flux $j$ regarding the $k_{2}$ parameter.

One may also assess the variation of the annual thermal loads according to variation of both thermal parameters of the brick layer. The thermal conductivity and the volumetric heat capacity are varied by $\pm 20 \%$ of their a priori values. Thus, their domains of variation are denoted as $k_{3} \in\left[0.8 \cdot k_{3}^{\circ}, 1.2 \cdot k_{3}^{\circ}\right]$ and $c_{3} \in\left[0.8 \cdot c_{3}^{\circ}, 1.2 \cdot c_{3}^{\circ}\right]$, respectively. The annual thermal loads on the interior surface are approximated using both the TAYLOR series expansion and the SRC linear expansion. Results are shown in Figure 7. Both approach gives similar results highlighting again the efficiency of the proposed method. One may also conclude that the variation the thermal loads is wider in the direction of the thermal conductivity rather than the volumetric heat capacity. It is consistent with previous results.

If both SRC and TAYLOR series expansion gives similar results for the annual thermal loads, it is not the case for the daily thermal loads of the insulation layer. Figure 8 gives the daily thermal loads during a summer day. It can be remarked that the variation of the thermal loads is strongly nonlinear with both input parameters. Thus, the SRC approach can not be ap-

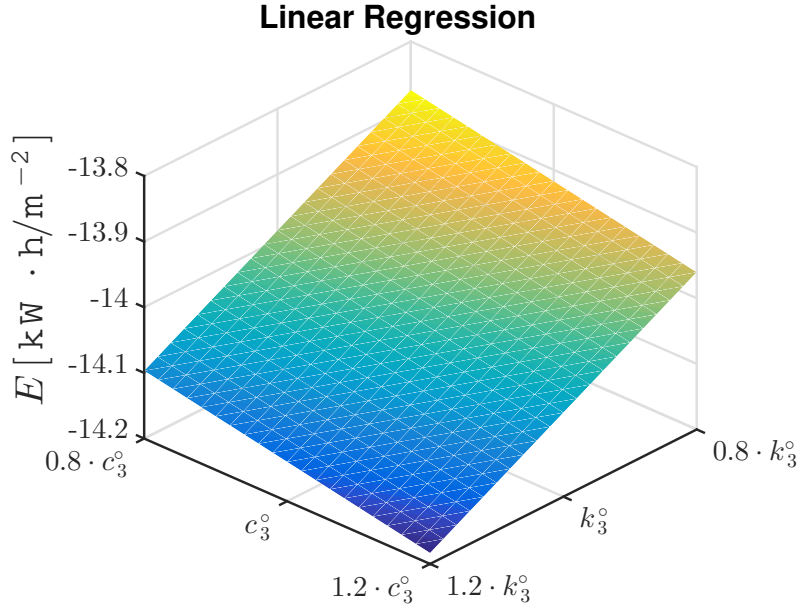

Taylor Expansion

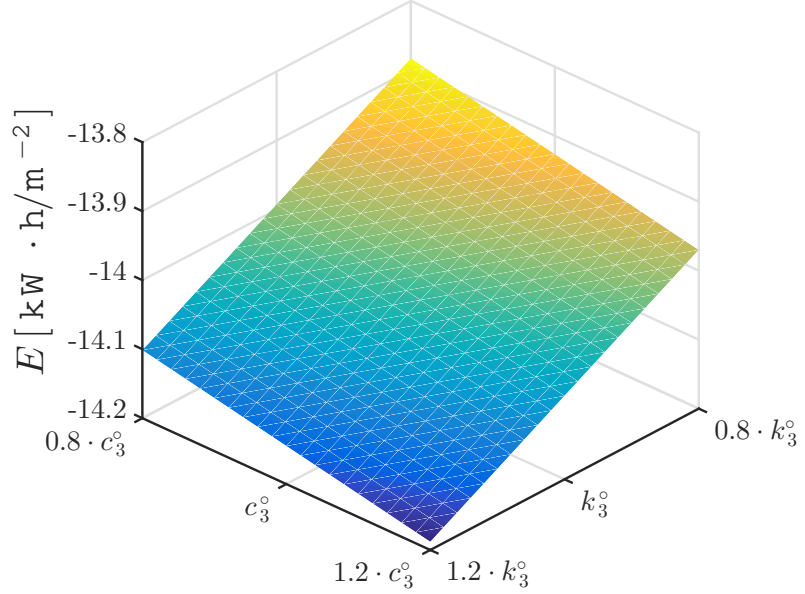

Figure 7: Extrapolation of the annual thermal loads $E$ according to $k_{3}, c_{3}$.

plied in such a case where the linearity hypothesis is not valid.

The monthly thermal loads on the inside surface are now studied. Their variations according to changes in the thermal conductivity parameter of the insulation layer are computed. The values of parameter $k_{2}$ are varied by $\pm 20 \%$ of the a priori value. The TAYLOR series approximation is used to calculate the expansion of the solution for the boundary values of $k_{2}=k_{2}^{\circ} \pm 0.2 k_{2}^{\circ}$. Figure 9 shows the expansion of the monthly thermal loads. They are negative almost all year since the heat flux is directed from the inside to the outside parts. Using this innovative approach, one may predict the evolution of the thermal loads with variation in the insulation material.

\section{Conclusion}

Implementation of the differential sensitivity analysis on the heat diffusion problem enables to retrieve continuous sensitivity coefficients. It allows exploring model outputs' variation according to changes in input parameters. In other words, it permits to identify the most influential parameters on any chosen model output. The derivative based approach has a 


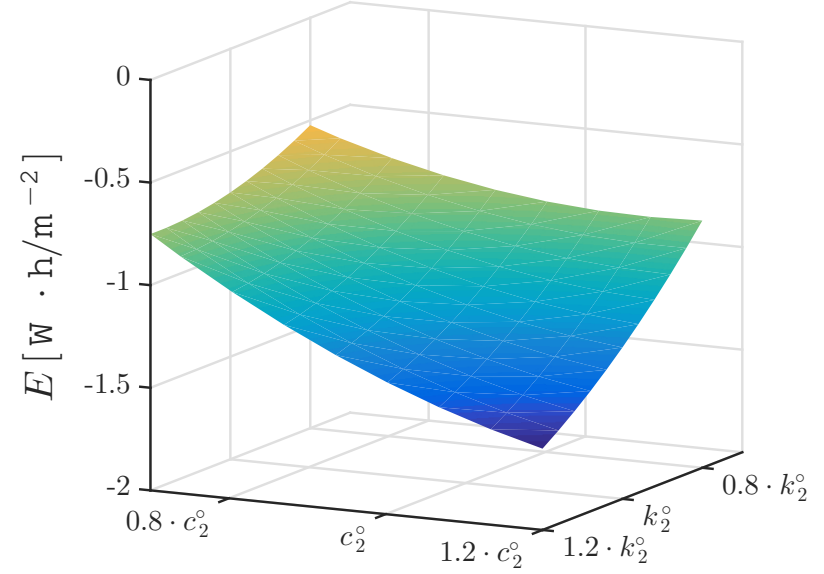

Figure 8: Extrapolation of the daily thermal loads $E$ accordina to $k_{\text {? }}, c_{?}$.

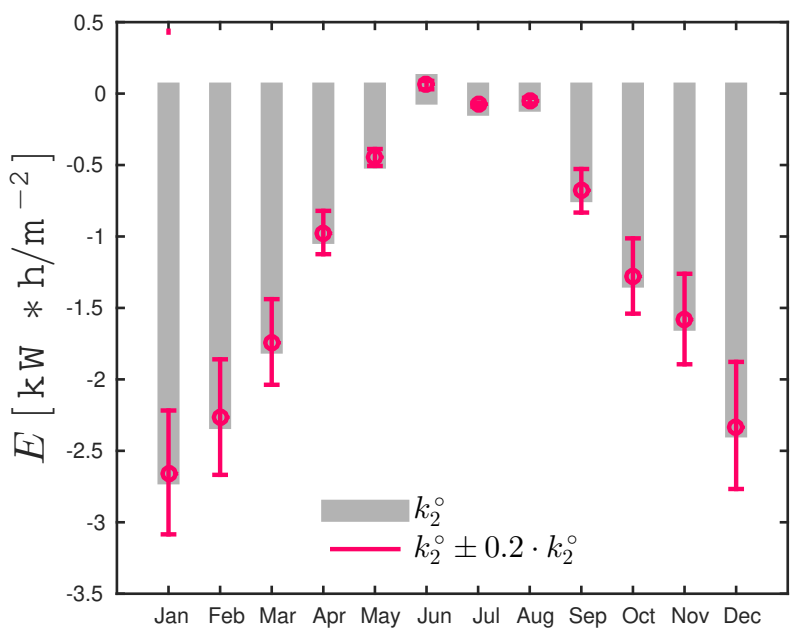

Figure 9: Monthly thermal loads and its variation according to changes in the parameter $k_{2}$.

comparatively smaller number of model evaluations. The innovative numerical model ensures accuracy and reliability of results with a low computational cost. Therefore, it can be used as a preliminary step for the parameter estimation problem. The proposed method can be extended to two or three-dimensional case study. It will require the solution of additional governing and sensitivity equations.

\section{Acknowledgment}

This work was partly funded by the "Conseil Savoie Mont Blanc" (CSMB), the French Atomic and Alternative Energy Center (CEA). The authors also acknowledge the Junior Chair Research program "Building performance assessment, evaluation and enhancement" from the University of Savoie Mont Blanc in collaboration with the French Atomic and Alternative Energy Center (CEA) and Scientific and Technical Center for Buildings (CSTB).

\section{References}

(2015). Annual Energy Outlook 2015, with Projections to 2040. Washington: EIA.
Barrio, E. P. D. and G. Guyon (2003). Theoretical basis for empirical model validation using parameters space analysis tools. Energy and Buildings 35(10), $985-996$.

Berger, J., S. Gasparin, D. Dutykh, and N. Mendes (2017). Accuracy of numerical methods applied to building energy performance.

Franchuk, A. (1969). Tables of heat properties of building materials. Stroyizdat. Moscow.

Gasparin, S., J. Berger, D. Dutykh, and N. Mendes (2018a, Jun). An improved explicit scheme for whole-building hygrothermal simulation. Building Simulation 11(3), 465-481.

Gasparin, S., J. Berger, D. Dutykh, and N. Mendes (2018b). Stable explicit schemes for simulation of nonlinear moisture transfer in porous materials. Journal of Building Performance Simulation 11(2), 129-144.

Kononovich, Y. (1986). Heating regime of buildings in mass construction. Stroyizdat. Moscow.

Mechri, H. E., A. Capozzoli, and V. Corrado (2010, October). USE of the ANOVA approach for sensitive building energy design. Applied Energy 87(10), 3073-3083.

Menberg, K., Y. Heo, and R. Choudhary (2016). Sensitivity analysis methods for building energy models: Comparing computational costs and extractable information. Energy and Buildings 133, $433-445$.

Ruiz Flores, R., S. Bertagnolio, and V. Lemort (2012, 07). Global sensitivity analysis applied to total energy use in buildings.

Saltelli, A., S. Tarantola, F. Campolongo, and M. Ratto (2004). Sensitivity Analysis in Practice: A Guide to Assessing Scientific Models. New York, NY, USA: Halsted Press.

Sun, Y., Y. Heo, M. Tan, H. Xie, C. J. Wu, and G. Augenbroe (2014). Uncertainty quantification of microclimate variables in building energy models. Journal of Building Performance Simulation 7(1), 17-32.

Trabelsi, A., Z. Slimani, and J. Virgone (2018). Response surface analysis of the dimensionless heat and mass transfer parameters of medium density fiberboard. International Journal of Heat and Mass Transfer 12\%, 623-630.

Wilde, P. and W. Tian $(2010,10)$. Predicting the performance of an office under climate change: A study of metrics, sensitivity and zonal resolution. Energy and Buildings, 1674-1684. 